\title{
Acquisizione dell'italiano accademico in contesto LS $e$ in prospettiva comparativa (modelli e analisi di abstract $e$ incipit)
}

\section{Acquisition of Academic Italian as a Foreign Language: The Comparative Perspective (models and analysis of abstracts and introductions)}

\author{
KATARÍnA Klimová [katarina.klimova@umb.sk] \\ Univerzita Mateja Bela v Banskej Bystrici, Slovacchia
}

\begin{abstract}
RiASSUNTO
Il contributo riguarda l'uso dell'inglese e dell'italiano nella comunicazione accademica e le questioni relative alla scrittura accademica in LS. In concreto, l'attenzione è indirizzata alle particolarità stilistiche della scrittura academica nelle due lingue, evidenziando similitudini e differenze nei due generi testuali del discorso accademico scritto: l'abstract e l'incipit dell'articolo scientifico. In quanto alla metodologia, la ricerca è basata sulla genre analysis e il corpus consiste di abstract e di introduzioni di articoli di ricerca relativi al settore delle scienze umanistiche, nello specifico della linguistica e della letteratura. Riteniamo il tema rilevante in relazione alla preparazione degli studenti universitari slovacchi di italiano L2 per la scrittura dei testi accademici in italiano.
\end{abstract}

\section{Parole Chiave}

Scrittura accademica; genre analysis; didattica; italiano

\begin{abstract}
This study deals with questions regarding the use of English and Italian in academic communication and the questions related to second language writing. It particularly draws attention to the stylistic particularities of academic writing in these two languages, pointing out some of their common and distinguishing features in two genres of academic discourse: the abstract and the introduction of a research article. As far as methodology is concerned, the research is based on genre analysis, and the corpus consists of abstracts and introductions of research articles from the humanities, specifically linguistics and literature. We believe that the knowledge of genre characteristics has the potential to help the students to write academic texts in their target contexts.
\end{abstract}

\section{KEYWORDS}

Academic writing; genre analysis; genre pedagogy; Italian language

RICEVUTO 2015-12-30; ACCETTATO 2016-03-01 


\section{Introduzione}

I processi di integrazione economica e politica che avvengono nel continente europeo dagli anni Cinquanta del XX secolo hanno portato con sé importanti cambiamenti anche nell'ambito dell'istruzione universitaria. Essi riguardano soprattutto l'armonizzazione dei sistemi educativi negli stati membri dell'UE, rafforzata ancor più dal Processo di Bologna che ha per obiettivo la formazione dello Spazio Europeo di Istruzione Superiore (EHEA). Il successo di questi progetti è correlato all'internazionalizzazione delle università ${ }^{1}$ che porta con sé la necessità di una comunicazione multilaterale. Nonostante il fatto che oggi sia l'inglese la lingua franca della comunicazione scientifica e accademica ${ }^{2}$, nelle università è presente oggi anche la volontà di preservare la diversità linguistica quale uno dei tratti costitutivi dell'Europa, come valore aggiunto e importante fattore di creatività ${ }^{3}$. Possiamo constatare che, nella situazione attuale, per studiosi, ricercatori e studenti universitari provenienti da paesi non-anglofoni, il pressuposto del loro lavoro è una condizione di bilinguismo o plurilinguismo a cui appartiene la conoscenza di norme discorsive locali. Questo è vero soprattutto nell'area delle scienze umanistiche in cui le lingue locali sono tuttora ben rappresentate. Questi settori - tra i quali rientra anche quello linguistico e linguistico-letterario, che è quello che ci interessa più da vicino - presentano così un interessante campo di studio delle specificità nazionali. Esaminandole si può osservare il loro più o meno marcato avvicinamento agli standard globali oggigiorno dettati dalla tradizione nordamericana della comunicazione accademica.

Nel presente contributo vogliamo dedicare attenzione ad alcuni aspetti relativi alla scrittura accademica in inglese e in italiano. Il tema ci interessa nell'ambito di un curricolo volto alla formazioni di studenti universitari di un corso di laurea in Italiano nel contesto slovacco o ceco ${ }^{4}$. Visto l'ambiente globale in cui le università sono inserite, abbiamo scelto di fare un confronto tra queste due lingue, inglese e italiano come lingue straniere (LS) di apprendenti di slovacco e ceco com L1. Abbiamo scelto la scrittura accademica per due motivi: primo perché la redazione dei testi accademici fa parte integrante dello studio universitario durante il quale lo studente dovrebbe acquisire le competenze necessarie per l'elaborazione di vari testi

1 Ovvero il processo di intensificazione della cooperazione come reazione alla sempre più forte concorrenza a livello globale, e allo stesso tempo come tentativo di un suo superamento attraverso una collaborazione più intensa nell'ambito dello scambio delle conoscenze, della diffusione delle innovazioni, dell'unificazione del potenziale di ricerca e delle risorse di finanziamento. L'internazionalizzazione viene realizzata attraverso il sostegno a programmi di scambio di docenti e di studenti, la creazione di percorsi di studio comuni e dottorati di ricerca congiunti e di comuni progetti didattici e di ricerca (cfr. per es. Alcón/Michavila 2012).

2 I termini comunicazione scientifica e comunicazione accademica sono spesso usati come sinonimi. Va però tenuto presente che l'espressione comunicazione scientifica può essere a volte utilizzata in senso stretto ovvero per indicare solo la comunicazione nell'ambito delle scienze dure (sotto l'influsso anglosassone); riferendosi invece all'ambito delle scienze molli, sarebbe preferibile usare comunicazione accademica o comunicazione scientifico-accademica quale termine che abbraccia ambedue i settori della comunicazione specialistica.

3 È stato dimostrato che gli individui o i gruppi plurilingui dispongono di un potenziale creativo più alto; cfr. per es. il Compendium (2009), Study on the Contribution of Multilingualism to Creativity, disponibile all'indirizzo http://eacea.ec.europa.eu/llp/studies/documents/study_on_the_contribution_of_multilingualism_to_creativity/compendium_part_1_en.pdf.

4 Sulle problematicità nel procedimento di ri-definizione del lessico specialistico della linguistica in esami di profitto scritti in contesto d'italiano LS (L1 slovacco) cfr. Nobili (in corso di stampa). 
accademici tra cui per es. riassunti, recensioni, elaborati di ricerca, tesi, tesine, dissertazioni; secondo, perché a questa attività non sempre viene dedicata sufficiente attenzione - i corsi di scrittura accademica riguardano per lo più la lingua inglese sia per l'importanza di questa lingua nonché per una percezione diversa dell'attività di scrittura in ambito culturale anglosassone ${ }^{5}$.

Il quadro in cui la nostra analisi è collocata è quello della genre analysis (Swales 1990, 2004) ${ }^{6}$. Essa prevede lo studio di un corpus rappresentativo di testi e l'identificazione di passaggi comunicativi di cui ogni genere è costituito. La linguistica diventa così lo strumento pratico che l'insegnante può utilizzare in classe facendo presente come vari modelli di vocabolario, grammatica e coesione strutturano i testi nei passaggi comunicativi che supportano l'obiettivo comunicativo di ogni genere.

In questo saggio evidenziamo dunque alcune particolarità dello stile accademico italiano rispetto a quello inglese analizzando due generi testuali: l'abstract e l'incipit dellarticolo scientifico. Abbiamo scelto questi due testi in quanto ritenuti esemplari, ma anche come testi con cui gli studenti durante il periodo dei loro studi si confrontano sia come fruitori sia come autori nella fase di scrittura di tesine, tesi e dissertazioni. In concreto, evidenziamo alcune tendenze nellorganizzazione retorica da parte di autori provenienti da diversi contesti culturali riassumendo e comparando gli esiti di due ricerche contrastive (inglese vs. italiano) precedentemente condotte (cfr. Klimová 2011 e 2014).

Il tema è altresì interessante perché permette di sviluppare, durante l'attività didattica, altre riflessioni, ad es., quelle sul concetto di genere e sulla sua applicazione nella didattica, sull'italiano contemporaneo di registro formale, sul linguaggio della scienza e sulla sua retorica ${ }^{7}$, sullo status dell'inglese nell'ambito delle comunità non anglofone e sul suo impatto sulle strategie comunicative nazionali. Anche se ci dedichiamo all'inglese e all'italiano come LS, la

5 In ambito culturale anglosassone la scrittura in generale, compresi i testi professionali o accademici, viene percepita come una competenza che può essere migliorata con la pratica, mentre, in ambito europeo, è piuttosto percepita come un'arte, un talento individuale (cfr. per es. Čmejrková 1994, 1997). Se guardiamo in concreto l'ambito ceco e slovacco, durante le scuole primarie e secondarie viene prestata attenzione alla scrittura di testi letterari o giornalistici; gli studenti iniziano i loro studi universitari senza alcuna preparazione alla scrittura di testi professionali e accademici che però vengono loro richiesti già dall'inizio (cfr. per es. Danišková 2014). Anche se hanno a disposizione vari manuali di scrittura professionale e accademica, in essi viene dedicata più attenzione agli aspetti formali e strutturali dell'elaborato rispetto alla sua efficacia comunicativa; i manuali che dedicano attenzione agli aspetti contenutistici sono ancora pochi, cfr. per es. per lo slovacco Vydra (2010); Staroňová (2011); per il ceco Čmejrková/Daneš/Světlá (1999). La situazione è analoga anche per l'italiano: cfr. per es. Cerruti/Cini (2007), Desideri/Tessuto (2011).

6 La genre analysis rappresenta negli ultimi vent'anni il paradigma dominante per gli studi del discorso accademico alla quale è stata dedicata attenzione anche dal punto di vista dell'insegnamento e dell'apprendimento di LS/L2. I tre approcci più significativi: (i) English for Specific Purposes (ESP) o English for Academic Puroposes (es. Swales, Bhatia, Hyland); (ii) Systemic Functional Linguistics (SFL) o Sydney School (es. Halliday, Martin) (iii) la Scuola della Nuova retorica (es. Bazerman, Berkenkotter, Huckin) si differenziano per un diverso approccio al testo nonché per l'applicazione della genre analysis alla didattica. Mentre la Nuova retorica nega la possibilità dell'insegnamento dei generi testuali affermando che essi subiscono troppo facilmente cambiamenti e sono fortemente dipendenti dal contesto, gli studiosi di ESP e SFL evidenziano invece la necessità di fornire agli studenti istruzioni esplicite su come va strutturato il testo e su quali sono le sue caratteristiche ed eventuali variazioni. Anche se ESP e SFL concettualizzano il genere in maniera diversa, ambedue cercano di rivelare lo schema retorico del genere e le sue caratteristiche chiave (cfr. per es. Hayland 2007).

7 Contrariamente alla percezione del linguaggio della scienza quale modalità oggettiva, neutrale ed antiretorica della comunicazione delle conoscenze, viene sempre di più messo in evidenza il suo carattere interattivo e la capacità comunicativa dell'autore del testo, su cui cfr. per es. Duszak (1997). 
consapevolezza di alcune particolarità di queste lingue affina la sensibilità per la riflessione linguistica e per la scrittura anche nella lingua materna o nelle altre lingue conosciute dallo studente.

\section{Metodologia}

Nell'ambito della genre analysis di Swales $(1990,2004)$, a partire dagli anni ottanta sono stati elaborati con obiettivi didattici modelli di riferimento per singoli generi testuali, es. abstract, articoli scientifici, monografie, grant proposals, recensioni, tesi, dissertazioni. L'attenzione si è rivolta soprattutto all'uso dell'inglese da parte di parlanti non nativi (il cosiddetto non-native o second language writing), che ha suscitato interesse per studi comparativi dell'inglese e di singole lingue nazionali o gruppi linguistici ${ }^{8}$. Ai generi testuali più elaborati nell'ambito della comunicazione scientifica e accademica appartengono l'abstract e l'incipit dell'articolo scientifico ${ }^{9}$.

L'abstract (nella tradizione italiana sommario, sintesi o riassunto) si è affermato, negli ultimi anni, come genere testuale autonomo, relativamente indipendente, internamente coerente e visibilmente strutturato (cfr. Van Dijk 1980), spazialmente collocato prima o dopo l'articolo scientifico $^{10}$. La sua funzione principale è di offrire al lettore, velocemente ed efficacemente, il contenuto di quello che vorrebbe o dovrebbe leggere, orientandolo così, spesso in anticipo, nel vasto contesto della produzione scientifica (Ventola 1994: 343).

L'incipit o l'introduzione a un saggio scientifico (article introduction) è invece una sua parte costitutiva. È la parte in cui l'autore presenta il tema e il modo in cui sarà trattato, contestualizza e giustifica i motivi della sua ricerca, mette in gioco la sua credibilità di studioso nei confronti di una determinata comunità discorsiva.

Si tratta dunque di due generi testuali strettamente relazionati che si prestano bene all'analisi essendo tra i più convenzionalizzati e, allo stesso tempo, testi relativamente brevi. Ambedue sono parti costitutive degli articoli scientifici e hanno un obiettivo comunicativo ben definito.

8 Gli studi contrastivi dei testi in L1 e L2 hanno una storia più lunga rispetto alla genre analysis. La tradizione della retorica contrastiva inizia con i lavori di Kaplan (1966). Lo studioso, basandosi sull'ipotesi del relativisimo linguistico di Sapir e Whorf, caratterizza la lingua come fenomeno culturale con proprie convenzioni retoriche. A partire dagli anni '80 il rinnovato interesse per gli studi di retorica contrastiva ha portato a ipotizzare l'esistenza di modelli culturalmente specifici di scrittura (cfr. es. Clyne 1987; Maurenan, 1993; Duszak 1994, 1997; Vassileva 2000).

9 Per l'abstract cfr. numerosi studi per la lingua inglese (es. Swales 1990, Swales/Feak 2009), per lo spagnolo (es. Martín-Martín 2002), per il francese (es. Van Bonn/Swales 2007), per il tedesco (es. Gnutzmann 1991), per lo svedese (es. Melander/Swales/Fredrickson 1997). Per l'incipit cfr. per es. per lo svedese (es. Fredrickson/Swales 1994), per il malese (es. Ahmad 1997), per il polacco (es. Golebiowski 1999), per lo spagnolo (es. Burgess 2002), per l'ungherese es. (es. Árvay/Tankó 2004), per il portoghese brasiliano (es. Hirano 2009).

10 Per quanto riguarda la terminologia, usiamo il termine articolo scientifico (ingl. scientific article) quale denominazione più generica di uno scritto rivolto a un pubblico di specialisti e pubblicato su una rivista specialistica o in atti di convegni. Esso viene considerato il genere testuale chiave della comunicazione scientifico-accademica anche se il suo peso varia nella gerarchia dei testi accademici a seconda degli ambiti di ricerca. Gode forse di maggior prestigio nelle scienze dure (dove per il suo carattere per lo più analitico e sperimentale viene denominato research article 'articolo di ricerca'; per le sue subcategorizzazioni cfr. Swales 2004: 213). Ad esso viene comunque riconosciuto un posto stabile (insieme ai libri e alle monografie) anche nell'ambito delle scienze socio-umanistiche, dove per il suo carattere retorico e saggistico viene spesso denominato saggio (ingl. essay). Ai fini del presente lavoro consideriamo sinonimi i termini articolo e saggio scientifico. 
Essi vengono considerati macrogeneri che presentano una subclassificazione interna dovuta alle differenze

(1) disciplinari, le cosiddette cross-disciplinary differences (scienze sociali, umanistiche, scientifico-naturali, economiche, tecniche, ecc.);

(2) nella funzione e nell'obiettivo comunicativo (abstract in riviste / abstract in abstracting journals /abstract per convegni; incipit nei saggi teorici o sperimentali);

(3) nel fattore socio-culturale, principalmente l'appartenenza linguistico-culturale dell'autore del testo.

Per quanto riguarda il primo punto ci concentriamo sulle scienze umanistiche, in particolare, sulla linguistica e sulla letteratura; per il secondo punto abbiamo analizzato gli abstract in riviste e gli incipit in atti del convegno; quanto al punto tre siamo partiti dalla convinzione che essendo l'atto di scrivere un atto sociale, gli studiosi hanno la tendenza a formulare i loro pensieri a seconda della propria nazionalità ed appartenenza a una certa comunità discorsiva.

Abbiamo basato la nostra analisi su due modelli che forse più hanno influenzato le riflessioni sugli abstract e sugli incipit sia nel mondo anglosassone come anche in successivi studi in altri paesi: il modello IMRD e il modello CARS ${ }^{11}$.

Il modello IMRD (Introduction, Method, Results, Discussion) è stato proposto per l'analisi della struttura retorica dei saggi empirici con una successione rigida delle loro parti costitutive, ed è stato applicato conseguentemente anche agli abstract che, essendo la sintesi del saggio, ne riproducono la struttura.

Il modello CARS (Create a Research Space) è stato invece proposto come struttura retorica per le introduzioni ai saggi (Swales 1990: 137-166). Esso dovrebbe rispecchiare lo sforzo di ogni autore per acquisire un proprio spazio nella ricerca e per essere accettato dalla comunità scientifica. Con l'avanzare degli studi questo schema è stato applicato anche agli abstract distinguendo così gli abstract informativi (più vicini alla struttura IMRD) da quelli indicativi (più vicina alla struttura CARS), cfr. Lorés (2004).

Ambedue i modelli si basano sulla segmentazione del testo in unità retoriche, le cosiddette moves 'mosse', suddivise a loro volta in steps, 'passi' (obbligatori e facoltativi) e sulla loro analisi secondo le intenzioni comunicative prototipiche di un genere. Riportiamo in tabella 1 i rispettivi schemi: lo schema IMRD e lo schema CARS (Swales 1990). Per questo ultimo riportiamo anche la sua versione aggiornata (Swales 2004):

11 Per quanto riguarda l'inglese ci si è basati sui risultati degli studi già esistenti per questa lingua. 


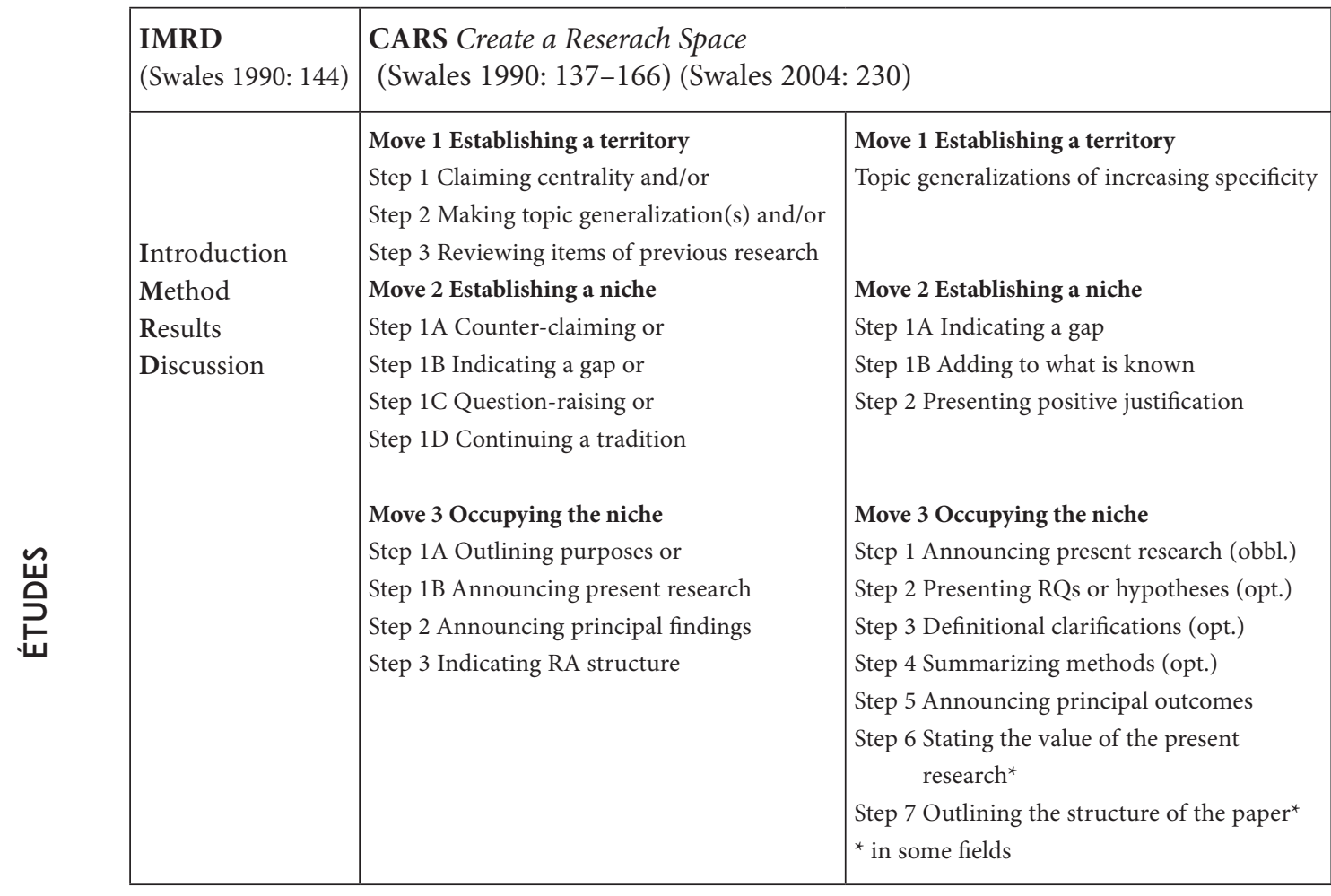

Tab. 1 Schema del modello IMRD e CARS

Abbiamo voluto verificare fino a che punto fosse possibile applicare tali modelli agli $a b$ stract e agli incipit scritti dagli studiosi italiani, e quali tendenze si potessero individuare nel settore linguistico e linguistico-letterario. Era prevedibile che i testi inseriti nel corpus non necessariamente corrispondessero ai modelli, dato il loro carattere per lo più teorico, la loro appartenenza alla macroarea umanistica e l'uso della lingua italiana. I presupposti di partenza per la comparazione interlinguistica sono stati due: (1) la definizione della nozione del genere testuale come riportata da Bhatia (2006: 88), il quale afferma che: "The most important aspect of a genre is that it is recognisable, sufficiently standardised and is based on a set of mutually accessible conventions, which most members of professional, academic or institutional organisations mutually share. [...] It is not static, fixed, or prescribed, but is often flexible, negotiated or sometimes contested". In questo senso le caratteristiche possono variare dal punto di vista disciplinare nonché culturale, ma il genere testuale conserva le sue caratteristiche di base; (2) La convinzione che nonostante la varietà delle opinioni sulla delimitazione esatta delle moves, sullo status degli steps, sulla sequenza e obbligatorietà delle moves e degli steps, la struttura renda possibile analizzare gli obiettivi comunicativi dell'autore e possa, come tale, servire da punto di partenza. 


\section{Il corpus}

\subsection{Gli abstract}

Come primo passo abbiamo esaminato la presenza o meno di abstract in dodici riviste (2000 - 2008). Di queste, cinque riviste contengono abstract in lingua inglese, due riviste contemporaneamente in inglese e in italiano e una rivista in tre lingue, inglese, italiano e francese; quattro riviste invece non mettono a disposizione gli abstract. I risultati si trovano nella tabella 2:

\begin{tabular}{|c|c|c|}
\hline & Lingua di abstract & Denominazione della rivista \\
\hline 1 & ING & Archivio Glottologico Italiano \\
\hline 2. & ING & Italian Journal of Linguistics \\
\hline 3. & ING & Rassegna Italiana di Linguistica Applicata \\
\hline 4 & ING & Studi Italiani di Linguistica Applicata \\
\hline 5. & ING & Studi e Saggi Linguistici \\
\hline 6. & ING + IT & Rivista Italiana di Onomastica \\
\hline 7. & ING + IT & Bollettino di Italianistica (abbr. BI) \\
\hline 8. & ING + IT + FR & Lingua Italiana d'Oggi \\
\hline 9. & 0 & Lingua e stile \\
\hline 10. & 0 & Studi di Lessicografia Italiana \\
\hline 11. & 0 & Studi Linguistici Italiani \\
\hline 12. & 0 & \\
\hline
\end{tabular}

Tab. 2 Gli abstract in alcune riviste italiane del settore linguistico e linguistico-letterario

Per gli scopi della ricerca sono state scelte due riviste italiane monolingue con circolazione nazionale con saggi sia di linguistica che di letteratura di autori italiani in italiani, acompagnati dai relativi abstract in italiano: Bollettino di Italianistica (BI) e Linguistica e letteratura (LL). In tutto sono stati esaminati 60 abstract (30 per ogni rivista). 


\subsection{Gli incipit}

Per quanto riguarda gli incipit, oggetto dell'analisi sono stati i testi pubblicati negli atti dei convegni. Anche per gli incipit il corpus è stato monolingue. Abbiamo esaminato 20 saggi pubblicati tra gli anni 2001-2008 da 20 diversi autori di madrelingua, membri affermati della comunità scientifica italiana. Tra i vari settori di ricerca in italianistica abbiamo scelto saggi tematicamente incentrati sulla relazione tra lingua e cultura, sulla linguistica storica e sulla glottodidattica. Hanno fatto parte del corpus sette saggi del volume Lingue e culture fra identità e potere; abbr. LC 1-7); sei del volume Formare nei paesi d'origine per integrare in Italia. Le nuove sfide della Dante Alighieri; abbr. DA 1-6); cinque saggi dal volume La sintassi dell' italiano antico, abbr. ItalAnt 1-5); due saggi tratti dal volume L'identità linguistica e culturale degli stranieri in Italia: insegnamento e acquisizione dell'italiano, abbr. ItalAnt 1-2). Si è selezionato il corpus in modo da veder garantita la maggiore omogeneità possibile per status del volume e lunghezza dei saggi. Nel corpus abbiamo scelto solo i saggi che dispongono di una chiara indicazione della parte iniziale.

\section{Dicussione}

Come prima osservazione si può affermare che i saggi nel corpus (come anche quelli che riguardavano gli abstract) hanno per lo più carattere teorico, perciò ambedue le tipologie tendono più verso il modello CARS. Abbiamo adoperato questa struttura retorica come modello di riferimento in base al quale è stato possibile individuare affinità e differenze nell'organizzazione delle principali unità retoriche. In generale possiamo affermare che è stata confermata una grande eterogeneità di scelte retoriche. Sia l'abstract sia gli incipit mostrano delle deviazioni dai rispettivi modelli presentando varie combinazioni dei principali passi retorici con risultati comunicativi diversi da quelli presenti nella cultura anglosassone.

Tra le tendenze più evidenti che siamo riusciti ad identificare negli abstract nonché negli incipit, che differenziano questi testi in modo netto dallo stile angloamericano, è l'indebolimento fino all'assenza della dimensione persuasiva. Dai testi si evince il relativo disinteresse dell'autore a "vendere" la sua ricerca nel vasto mercato della produzione scientifica. Gli autori non sentono il bisogno di contestualizzare la ricerca né di giustificarne la sua importanza. Vogliamo evidenziare queste tendenze con esempi concreti.

4.1. Negli abstract l'attenzione degli autori è dedicata piuttosto all'indicazione generale del tema del saggio (BI 53,3\%, LL 30\%) e della struttura del saggio e/o ai principali risultati. Gli abstract hanno perciò carattere di semplice resoconto dei punti più importanti trattati nel contributo $^{12}$. In questo senso, il modo in cui gli autori presentano la loro ricerca è molto simile a quello francese descritto da Van Bonn/Swales (2007: 98); i due studiosi affermano che gli abstract inglesi esprimono "Here is why this research is important", mentre quelli francesi "Here is what this research attempts to do"; cfr. es. (1):

12 Le percentuali sono le seguenti: gli abstract con l'indicazione diretta del tema (BI 53,3\%, LL 30\%), gli abstract con un'affermazione preliminare all'indicazione del tema del saggio (BI 16,6\%, LL 23,3\%), gli abstract con un'affermazione della centralità o dell'importanza dell'argomento (BI 13,3\%, LL 26,6\%), gli abstract con un'affermazione dell'obiettivo della ricerca (BI 6,6\%, LL 13,3\%), gli abstract che iniziano in medias res (BI 6,6\%, LL 6,6\%). 
(1) tema La rassegna che ci si offre sull'italiano regionale affronta i principali nodi teorici della discussione scientifica sull'argomento. indicazione della struttura del saggio In modo particolare vengono affrontati il problema del rapporto tra le varietà regionali e le altre dimensioni della variazione, la maggiore o minore permeabilità dei diversi livelli d'analisi all'interferenza con il dialetto (con particolare attenzione al lessico e alla fraseologia), alla delimitazione geografica delle varietà regionali e il prestigio di ciascuna di esse. Si è cercato, inoltre, di fornire un quadro anche delle nuove e/o auspicabili linee di ricerca, soffermandosi più approfonditamente sulla percezione del parlante riguardo alla variazione regionale dell'italiano. (BI/2008)

Dalla nostra analisi è emerso che agli abstract non viene ascritto lo stesso peso che nella cultura anglosassone. In base alla tipologia dei riferimenti metatestuali, abbiamo riscontrato che gli abstract funzionano piuttosto come unità indipendenti (cfr. le tipiche costruzioni inglesi con il dimostrativo this paper, in the present contribution vs. costrutti italiani con l'articolo definito: larticolo presenta, il saggio affronta, il contributo prende in esame) che danno l'impressione di un maggior distacco dal testo che accompagnano. Mentre nella cultura angloamericana l'abstract si è affermato come parte essenziale dell'articolo scientifico, sembra che gli autori italiani riservino ad esso una funzione piuttosto marginale. Quest'impressione è ancora più accentuata dalla sovente collocazione degli abstract alla fine della rivista. Questa condizione può essere determinata dal fatto che quella italiana è una comunità scientifica relativamente circoscritta, dei cui membri le ricerce, almeno nelle loro linee essenziali sono note.

4.2. Gli incipit dimostrano tratti analoghi. Seguendo sempre il modello CARS, la M1 (cfr. tab. 1) è la move meno realizzata. Nei casi di realizzazione, gli autori ricorrono più spesso allo step 2 dando informazioni generali sull'argomento (7 casi). Gli autori sottolineano la frequenza o la complessità dell'argomento o il fatto che la ricerca da loro riportata rientri in un settore di studi riconosciuto che fa parte di tematiche condivise dalla comunità scientifica; cfr. es. (2):

(2) Il plurilinguismo, come è noto, è patrimonio costitutivo stesso della storia della nostra penisola. (DA1)

Per segnalare eventuali lacune (obiettivo della M2), negli incipit generalmente si evita una critica o una polemica aperta con gli autori coinvolti nella ricerca sullo stesso tema. Eventuali "punti deboli" negli studi fino a quel momento condotti vengono segnalati in modo generico: si evidenzia l'assenza di un'analisi più approfondita (ess. 3, 4) o l'assenza di studi al riguardo per la novità del fenomeno, soprattutto nei saggi che trattano temi di cambiamenti sociali recenti.

(3) Alla genesi di queste costruzioni non è stata prestata particolare attenzione, forse anche a causa della loro assenza in Dante e Petrarca. (ItalAnt3)

(4) Mentre in altri paesi la ricerca in questo campo si trova già a uno stadio avanzato [...], in Italia si è scontato un certo ritardo. [...] Se, dunque, per quel che concerne la descrizione sincronica dell'italiano contemporaneo, questa impostazione sembra essersi affermata [...], è ancora 
tutta da realizzarsi l'opera di analisi e descrizione delle valenze dell'italiano antico, intendendo con ciò sia ciascuno degli stadi sincronici isolabili in base a criteri valenziali nella lingua del passato, sia l'evoluzione diacronica da uno stadio all'altro. (ItalAnt4)

Sia negli abstract che negli incipit (nell'ambito della M3) si preferisce comunicare al lettore gli obiettivi della ricerca o si annuncia il tema (senza sottolineare perché questo tema abbia importanza). In due casi gli incipit consistono solo della M3 (nella maggioranza dei casi realizzata attraverso lo step $1 \mathrm{~A}$ e $1 \mathrm{~B}$ ). In tre introduzioni è stato riscontrato, in aggiunta, lo step 3 (indicating RA structure):

(5) Inizierò illustrando brevemente le ripercussioni che i cambiamenti nella popolazione scolastica della scuola d'obbligo - [...]- hanno determinato nelle richieste che si fanno oggi agli insegnanti. [...] Alla luce di tali richieste prenderò in considerazione alcune modalità formative possibili ed indicherò il ruolo che le università possono - o dovrebbero - assumere nella professionalizzazione degli insegnanti in servizio. Presenterò poi brevemente il secondo dei due corsi, da poco conclusosi. (II1)

Dalle tendenze accennate si evince che i due modelli di Swales (1990, 2004), IMRD e CARS, non sono come tali applicabili ai generi testuali analoghi in italiano, e ciò è in linea con le tendenze in altre lingue europee. Le maggiori differenze retoriche riflettono il fatto che la cultura nordamericana è una cultura fortemente competitiva e il modello CARS, elaborato in base al corpus dei saggi in inglese, mette in risalto proprio quest'alto livello di competizione per la promozione.

Per quanto riguarda gli incipit in italiano, sembra che sarebbe più adeguato attenersi a una struttura che tenga conto delle specificità nazionali. Swales (2004: 243-246), raccogliendo proposte da vari autori non anglofoni, propone ancora un altro modello, il cosiddetto OARO (Open a Research Option model) che, rispetto a quello CARS, riflette una minore competizione al di fuori della comunità anglossassone e un minore antagonismo. Il modello riportato in tabella 3 è più facilmente applicabile alle scienze molli presentando più variazioni possibili delle sue sezioni costitutive.

Modello OARO (Open a Research Option Model)

cfr. Swales 2004: 243-246

0 [Attracting the Readership] Optional opening (Fredrickson \& Swales, 1994)

1 Establishing Credibility (uno o più dei seguenti passi)

a. Sharing background knowledge (Golebiowski 1999)

b. Justifying need for research per se (Ahmad 1997)

c. Presenting interesting thoughts (Clyne 1985)

d. Introducing general goal (Golebiowski 1999)

2 Offering a Line of Inquiry

a. Discussing current problems

b. Expressing interest in an emerging topic

3 Introducing the topic

Tab. 3 Schema del modello OARO 
Sarebbe interessante riuscire a scoprire una tendenza tipicamente italiana per i vari settori di studio. Per le piccole dimensioni del corpus ciò non è stato possibile e si potrebbe rimandare ad uno studio futuro su un corpus più ampio.

\section{Conclusioni}

Concludendo, possiamo affermare che una parte considerevole dell'istruzione universitaria consiste nell'acquisizione della capacità di interpretare e produrre testi e che la comunicazione accademica si realizza in un ambiente relativamente stabile e delimitato dai precisi generi testuali. Nel saggio abbiamo voluto evidenziare alcune differenze concrete nell'impostazione dei due generi testuali dovute alla differenze linguistiche e culturali degli autori. La conoscenza di questo tipo di differenze è imprescidibile per studenti, dottorandi e ricercatori che, grazie ai numerosi programmi di scambio e di cooperazione internazionale, si muovono in un ambiente multiculturale e plurilingue.

Nonostante le norme sviluppate per la lingua inglese possano sembrare troppo rigide e contrarie allo stile individuale dell'autore del testo, siamo dell'opinione che esse possano essere d'aiuto per i principianti nella loro prima fase d'apprendimento della strutturazione del testo in una lingua straniera nonché in una fase successiva in cui va prestata attenzione anche alle norme vigenti in vari ambiti disciplinari e socioculturali. La conoscenza della differenziazione dei generi testuali in sistemi sociocultuali diversi rende più sensibili e consapevoli delle differenze dovute all'influenza del sostrato al momento dello scrivere in una lingua straniera ed aiuta ad innalzare la qualità dei testi prodotti.

\section{Riferimenti bibliografici}

Ahmad, K. Ummul. (1997). Research article introductions in Malay: Rhetoric in an emerging research community. In A. Duszak (Ed.), Culture and Styles of Academic Discourse (pp. 273-303). Berlin - New York: Mouton de Gruyter.

Alcón, E., \& Michavila, F. (2012). La universidad multilingüe. Madrid: Tecnos.

Árvay, A., \& Tankó, G. (2004). A contrastive analysis of English and Hungarian theoretical research article introductions. IRAL, 42, 1, 71-100.

Bhatia, Vijay K. (2006). Analysing genre: some conceptual issues. In M. Hewings (Ed.), Academic writing in context. Implications and applications (pp. 79-92). London: Continuum.

Burgess, S. (2002). Packed houses and intimate gatherings: Audience and rhetorical structure. In J. Flowerdew (Ed.), Academic discourse (pp.196-215). Harlow: Longman.

Cerruti, M., \& Cini, M. (2007). Introduzione elementare alla scrittura accademica. Roma-Bari: Laterza.

Clyne, Michael. (1987). Cultural Differences in the Organization of Academic Texts: English and German. Journal of Pragmatics, 11, 211-247.

Čmejrková, S. (1994). Non-native (academic) writing. In S. Čmejrková et al. (Eds.), Writing vs. Speaking. Language, Text, Discourse, Comunication (pp. 303-310). Tubingen: Narr. 
Čmejrková, S., \& Daneš, F. (1997). Academic writing and cultural identity: the case of Czech academic writing. In A. Duszak (Ed.), Culture and styles of academic discourse (pp. 41-61). Berlin - New York: Mouton de Gruyter.

Čmejrková, S., Daneš, F., \& Světlá, J. (1999). Jak napsat odborný text. Praha: Leda.

Danišková, Z. (2014). Učit písat študenta vysokej školy? Potenciál kurzu akademického písania. Orbis Scholae, 8, 1, 129-143.

Desideri, P., \& Tessuto, G. (2011). Il discorso accademico. Le lingue pratiche e disciplinari. Urbino: Quattroventi.

Duszak, A. (1994). Academic discourse and intellectual styles. Journal of Pragmatics, 21, pp. 291-313.

- (1997). Culture and Styles of Academic Discourse. Berlin - New York: Mouton de Gruyter.

Fredrickson, K. M., Swales, J. M. (1994). Competition and discourse community: introductions from Nysvenska Studier. In B.-L. Gunnarsson et al. (Eds.), Text and Talk in Professional Contexts (pp. 9-22). Uppsala: Uppsala university.

Golebiowski, Z. (1999). Application of Swales' model in the analysis of research papers by Polish authors. International Review of Applied Linguistics, 37, 231-47.

Gnutzmann, C. (1991). “Abstracts" und "Zusammenfassungen” im deutsch-englischen Vergleich. Das Passiv als interkulturelles und teiltextdifferenzierendes Signal. In B.-D. Müller (Ed.), Interkulutrelle Wirtschaftskommunikation (pp. 363-378). München: Iudicium Verlag.

Hirano, E. (2009). Research article introductions in English for specific purposes: A comparison between Brazilian Portuguese and English. English for Specific Purposes, 28, 4, 240-250.

Hyland, K. (2007). Genre pedagogy: Language, Literacy and L2 writing instruction. Journal of Second Language Writing Instruction, 16, 148-164.

Kaplan, R. (1966). Cultural thought patterns in intercultural education. Language Learning, 16, 9-22.

Klimová, K. (2011). L'abstract tra funzionalità e pragmatismo nell’odierno paesaggio comunicativo (con particolare attenzione all'italiano). In G. Held \& S. Schwarze (Eds.), Testi brevi: teoria e practica della testualità nellera multimediale (pp. 281-296). Frankfurt am Main: Peter Lang.

Klimová, K. (2014). L“italianità” nell'incipit dei saggi scientifici di ambito linguistico. In M. Arcangeli (Ed.), .LId'O: lingua italiana d’oggi IX-2012 (pp. 193-206). Roma: Bulzoni Editore.

Lorés, R. (2004). On RA abstracts: from rhetorical structure to thematic organization. English for Specific Purposes, 23, 280-302.

Martín-Martín, P. (2002). A genre based investigation of abstract writing in English and Spanish. Revista Canaria de Estudios Ingleses, 44, 47-64.

Maurenan, A. (1993). Cultural differences in academic rhetoric. A textlinguistic study. Frankfurt am Main Berlin - Bern - New York - Paris: Peter Lang.

Melander, B., \& Swales, J. M., \& Fredrickson, K. M. (1997). Journal abstracts from three academic fields in the United States and Sweden: national or disciplinary proclivities? In A. Duszak (Ed.), Culture and Styles of Academic discourse (pp. 251-272). Berlin - New York: Mouton de Gruyter.

Nobili, C. (in stampa). Scrivere per farsi capire e valutare. Analisi di un corpus di esami di studenti universitari slovacchi in contesti di italiano LS. In Perchè scrivere: motivazioni, scelte, risultati. Franco Cesati Editore: Firenze.

Staroňová, K. (2011). Vedecké písanie. Martin: Osveta.

Swales, J. M. (1990). Genre Analysis. English in academic and research settings. Cambridge: Cambridge University Press.

. (2004). Research genres: Explorations and applications. Cambridge: Cambridge University Press.

Swales, J. M., \& Feak, Ch. B. (2009). Abstracts and the Writing of Abstracts. Michigan: The University of Michigan Press. 
Van Bonn, S., \& Swales, J. M. (2007). English and French journal abstracts in the language sciences: Three exploratory studies. Journal of English for Academic Proposes, 6, 93-108.

Van Dijk, T. A. (1980). Macrostructures. Hillsdale, NJ: Erlbaum.

Vassileva, I. (2000). Who is the author? (A contrastive analysis of authorial presence in English, German, French, Russian and Bulgarian academic discourse). Sankt Augustin: AsgardVerlag.

Ventola, E. (1994). Abstracts as an object of linguistic study. In S. Čmejrková et al. (Eds.), Writing vs. Speaking. Language, Text, Discourse, Comunication (pp. 333-352).Tübingen: Narr.

Vydra, A. (2010). Akademické písanie. Ako vzniká filozofický text. Trnava: Filozofická fakulta Trnavskej univerzity v Trnave. 


\title{
Corpus
}

\begin{abstract}
:
Linguistica e letteratura. Rivista semestrale diretta da Roberto Mercuri Pisa - Roma: Fabrizio Serra editore. Bollettino di italianistica. Rivista semestrale di critica, storia letteraria, filologia e linguistica, diretta Alberto Asor Rosa, Roma: Carocci.
\end{abstract}

Incipit:

Arcangeli, M., \& Marcato, C. (eds). (2008). Lingue e culture fra identità e potere. Bonacci: Roma.

(LC1) Cortelazzo, M. A., "I discorsi di fine d'anno dei Presidenti della Repubblica. Il lessico" (13-24).

(LC2) Caraffi, P., "Christine Pizan. La politica e la guerra” (101-109).

(LC3) Mariottini, L., “www.identità/alterità.com” (157-168).

(LC4) Marra, A., "Identità e diversità nello slavo del Molise. Appunti sul sistema nominale" (201-213).

(LC5) Di Filippo, M., "Considerazioni sull'uso dei gallicismi nel romanzo 'Lo Straniero' di Ivan Šmelev" (267-278).

(LC6) Massariello Merzagora, G. "Lingue e gerarchie delle lingue nei lager nazisti” (481-491).

(LC7) Franceschini, F., "Rapporti di potere, identità, lessico nella Venezia di Livorno (percosse, sesso, gergo, imprecazioni)” (509-522).

Arcangeli, M., \& Masi, A. (eds). (2006). Formare nei paesi dorigine per integrare in Italia. Le nuove sfide della Dante Alighieri. Roma: Società Dante Alighieri.

(DA1) Arcangeli, M., "Integrazione e formazione nell'Italia neoplurilingue" (55-66).

(DA2) Morcellini, M., "Mediare il culturalismo. Quale ruolo per formazione, comunicazione e classi dirigenti” (67-84).

(DA3) Remotti, F., "La comunicazione interculturale - dialoghi tra 'noi' incompleti” (85-102).

(DA4) Scaglione, S. "Diritti umani linguistici e diritti linguistici dei migranti” (103-121).

(DA5) Menzinger, C., "La formazione dei docenti e la metodologia didattica" (143-158).

(DA6) Russo Spena, M., "La dimensione culturale nella preformazione lingusitica a futuri immigrati: il caso Tunisia” (169-178).

Dardano, M., \& Frenguelli, G. (eds.). (2004). SintAnt. Lasintassi dellitaliano antico. Atti del Convegno internazionale di studi (Università di “Roma Tre", 18-21 settembre 2002). Roma: Aracne.

(ItalAnt1) Librandi, R., “Tratti sintattico-testuali e tipologia di testi: la trattatistica scientifica” (271-292).

(ItalAnt2) Lauta, G., "Sui verbi introduttivi del discorso riportato nell'italiano antico” (253-269).

(ItalAnt3) Palermo, M., "Le perifrasi imminenziali in italiano antico" (323-349).

(ItalAnt4) Poggiogalli, D., "Alternanze strutturali in verbi trivalenti: prime esplorazioni” (373-390).

(ItalAnt5) Renzi, L., "L'articolo in italiano antico" (391-407).

Scaglione, S. (ed.). (2004). L'identità linguistica e culturale degli stranieri in Italia: insegnamento e acquisizione dellitaliano. Roma: Bulzoni.

(II1) Ciliberti, A., "La professionalizzazione degli insegnanti in contesti multilingui: riflessione ed azione" (91-112).

(II2) Sinopoli, F. "Letteratura e migrazione in Italia. La banca dati Online Basili sugli scrittori immigrati e sulle loro opere in lingua italiana" (213-224). 